\title{
Introduction to Panel Discussions at the Third International Conference on Open Source Systems - OSS 2007
}

\author{
Sandra A. Slaughter ${ }^{1}$ \\ 1 David A. Tepper School of Business, Carnegie Mellon University, \\ Pittsburgh, Pennsylvania, U.S.A. email: sandras@andrew.cmu.edu, web: \\ http://www.tepper.cmu.edu/andrew/sandras
}

\section{Summary}

Two diverse, stimulating and important panel discussions are included in this year's programme on open source systems.

The first panel explores the diverse set of legal issues and risks that arise as governments increasingly adopt open source systems. Governments are attracted to open source software systems by the potential cost savings, open standards and protocols, and the flexibility, adaptability and reliability of the systems. However, there are serious legal issues and liabilities that can arise when governments adopt open source systems. This panel identifies and examines potential legal risks and liabilities from open source systems adoption in the government context. Some of the important legal concerns discussed by this panel include intellectual property rights, licensing, and protection of sensitive or confidential information.

The second panel is organized as a debate in which the panelists will consider the advantages and disadvantages of the sharing of research data and analyses by open source systems researchers. The area of open source systems has attracted numerous researchers from many different disciplines. In part, the attraction of research on open source systems is due to the public information available about open source communities. Using this information, researchers have been uncovering fascinating insights about how open source systems are developed and used and how open source communities function. Unfortunately, as noted by this panel, the work products of this research are not readily available to the public. This prevents new researchers from validating, building upon and extending the research already conducted by others. However, making research work products available to the others, while potentially valuable, has associated disadvantages. For example, certain kinds of data collected by a researcher (such as surveys or other confidential information) may not be released to the public, given human subjects concerns. In

Please use the following format when citing this chapter:

Slaughter, S.A., 2007, in IFIP International Federation for Information Processing, Volume 234, Open Source Development, Adoption and Innovation, eds. J. Feller, Fitzgerald, B., Scacchi, W., Sillitti, A., (Boston: Springer), pp. 351-352. 
addition, it can require substantial cost and effort on the part of the researcher to make research work products understandable and usable by others. This panel debates the issues surrounding the pros and cons of making open source systems research work products available to researchers. 